Pacific Journal of Mathematics

WELL-ORDERED SUBSETS AND MAXIMAL MEMBERS O 


\title{
WELL-ORDERED SUBSETS AND MAXIMAL MEMBERS OF ORDERED SETS
}

\author{
HERBERT E. VAUGHAN
}

1. Introduction. Kuratowski [2] and Milgram [3] have given procedures for avoiding the use of transfinite numbers in the proofs of many theorems. The procedure of Kuratowski is based on results of Hessenberg [1] concerning the existence of well-ordered subsets of ordered sets, and Milgram's is based on a similar theorem [3, p. 23, Th. 1']. Although Milgram's theorem does not strictly include Hessenberg's results, his procedure is more generally applicable than is Kuratowski's. Milgram has given two proofs of his theorem, of which the first (and simpler) utilizes transfinite numbers; the second, like Hessenberg's, avoids their use by methods similar to those used by Zermelo in his second proof of the well ordering theorem [6]. Szele [4] has given a proof, patterned on Zermelo's first proof of the well ordering theorem [5], that Zorn's lemma is a consequence of the axiom of choice.

The purpose of this note is to show that Szele's proof can be modified in such a way as to prove a common generalization (Theorem 1) of the theorems of Hessenberg and Milgram. The proof is as simple as Milgram's first but, like his second, makes no use of ordinal numbers. Furthermore, it can be arranged in such a manner that Szele's theorem appears as an intermediate result almost as simply as in [4].

2. A general result. We shall use the following notations. The relation < orders $M$ if it is transitive: $a<b$ and $b<c \Rightarrow a<c$; $n$ is an upper (lower) bound of $N$ if $p \leq n(n \leq p)$ for every $p \in N ; n$ is a greatest (least) member of $N$ if it is an upper (lower) bound of $N$ and belongs to $N ; m$ is a maximal member of $M$ if $m<n \Longrightarrow n<m$ for every $n \in M$; a subset of $M$ will be said to be bounded if it has an upper bound; the symbol ' $C$ ' will denote proper inclusion, and ' $\Lambda$ ' will denote the empty set.

The relation < well orders $N$ if every nonempty subset of $N$ has a unique least member. If this is the case then $<$ is transitive, antisymmetric: $a<b$ and $b<a \Longrightarrow a=b$, and connected: $a \neq b \Longrightarrow a<b$ or $b<a$, on $N$. If $n \in N$ 
then $N_{n}=\{x \mid x \in N$ and $n \Varangle x\}$, and if $n$ is not the greatest member of $N$ then $n^{+}$is the least member of $\{x \mid x \in N$ and $x \notin n\}$. The greatest member of $N_{n}+$ is $n$.

THEOREM 1. Let $M$ be ordered by $<, a \in M$, and $u$ a function which assigns an upper bound to every bounded subset of $M$ in such a way that: if $N, P \subseteq M$ and have a common greatest member then $u(N)=u(P)$; while if $\Lambda \subset N, P \subseteq M$, and neither has a greatest member but both have the same nonempty set of upper bounds, then $u(N)=u(P)$; and, finally, $u(\Lambda)=a$. Let $\eta$ be the class of all sets $N \subseteq M$ each of which is well ordered by < and has the property that, for every $n \in N$, we have $n=u\left(N_{n}\right)$. Let $P$ be the class of all sets $P \subseteq M$ each of which has the property that, for every bounded set $N \subseteq P$ which contains no upper bound of $P$, we have $u(N) \in P$, while if $N \subseteq P$ and contains an upper bound $m$ of $P$, we have $u(N) \leq m$. Then the greatest member of $\eta$ and the least member of $P$ exist and are the same set, $M(u)$. The least member of $M(u)$ is $a$, and if $M(u)$ is bounded it has a greatest member $m$, and $u(M(u)) \leq m$. A necessary and sufficient condition that an $m \in M(u)$ be the greatest member of $M(u)$ is that $u(\{m\}) \leq m$.

REMARKS. If $<$ is antisymmetric then $P$ is the class of all sets $P \subseteq M$ such that, for each bounded set $N \subseteq P$, we have $u(N) \in P$. Under the same hypothesis we have: If $M(u)$ is bounded, then $u[M(u)]$ is its greatest member; and an $m \in M(u)$ is its greatest member if and only if $u(\{m\})=m$.

Milgram's theorem is a consequence of the theorem obtained from the above by requiring that $<$ be irreflexive and that $u(N)=u(P)$ whenever $N$ and $P$ have the same nonempty set of upper bounds, and by removing the requirement that $u(\Lambda)=a$. Theorem 1 then generalizes only the special case of Milgram's theorem which is obtained by adjoining ' $u(\Lambda)=a$ ' to the hypotheses of the latter. However, this normalization places no restriction on the applicability of Theorem 1 and is introduced merely to simplify the statement and proof of this theorem. Hessenberg's results follow from the special case of Theorem 1 which is obtained by requiring that < be reflexive and antisymmetric, that if $\Lambda \subset N \subseteq M$ then sup $N$ exists, and that there is a transformation $f$ of $M$ in itself such that for every $m \in M$ we have $m<f(m)$, and in terms of which $u$ is defined as follows: For each $N \subseteq M$ which has a greatest member $n$ we have $u(N)=f(n)$, while if $\Lambda \subset N \subseteq M$ and $N$ has no greatest member, then $u(N)=\sup N$.

3. Proof of Theorem 1. There exist nonempty sets which are members of $\eta$, for example $\{a\}$, and $a$ is the least member of every such set. If $N, P \in \cap$ then one is a segment of the other. For if $N_{n}=P_{p}$ is a common proper segment of $N$ and $P$ then $n=u\left(N_{n}\right)=u\left(P_{p}\right)=p$, and $N_{n} \cup\{n\}=P_{p} \cup\{p\}$ is a larger 
common segment of $N$ and $P$. Hence the greatest common segment of $N$ and $P$ (which exists, since it is merely the join of all common segments) is not a proper segment of both of them and so must be either $N$ or $P$. It follows that the join, $M(u)$, of all members of $\eta$ is a member of $\eta$. If $M(u)$ is bounded and $u[M(u)] \in M(u)$ then $u[M(u)]$ is the greatest member of $M$. If $u[M(u)] \notin$ $M(u)$ then $M(u) \cup\{u[M(u)]\} \not \ell$. This can only be because, for some $m \in$ $M(u)$, we have $u[M(u)] \leq m$. Since < is transitive, $m$ is the greatest member of $M$.

If $m \in M(u)$ and is not the greatest member of $M(u)$ then, since $m$ is the greatest member of $M(u)_{m}{ }$, we have $u(\{m\})=u\left[M(u)_{m^{+}}\right]=m^{+} \Varangle m$.

Suppose $N \subseteq M(u)$ and is bounded. Then $N$ has an upper bound in $M(u)$. For if this were not the case then every upper bound of $N$ would be an upper bound of $M(u)$ and the latter would have an upper bound, and consequently a greatest member, contradicting the assumption that $N$ has no upper bound in $M(u)$.

Now let $n$ be the least upper bound of $N$ in $M(u)$. If $n \in N$ then either $n$ is the greatest member of $M(u)$ and $u(N)=u(M(u)) \leq n$, or $u(N)=u\left(M(u)_{n^{+}}\right)=$ $n^{+} \in M(u)$. If $n \notin N$ then either $N=\Lambda$ and $u(N)=a \in M(u)$, or $\Lambda \subset N$ and neither $N$ nor $M(u)_{n}$ has a greatest member but both have the same upper bounds and $u(N)=u\left[M(u)_{n}\right]=n \in M(u)$. Hence $M(u) \in P$.

Finally, if $P \in P$ then $M(u) \subseteq P$. For suppose that $m \in M(u)$ such that $M(u)_{m} \subseteq P$. Then $M(u)_{m}$ cannot contain an upper bound of $P$. For if $n$ were such an upper bound then, since $P \in P$, it would follow that $m=u\left[M(u)_{m}\right] \leq n$, contradicting the fact that, since $n \in M(u)_{m}$, we have $m \Varangle n$. Hence if $M(u)_{m} \subseteq P$ then $m=u\left[M(u)_{m}\right] \in P$. It follows by induction that $M(u) \subseteq P$.

4. Corollaries. We note the following results.

COROLLARY 1. Let $u$ satisfy the additional restriction that any $m \in M$ which is not maximal satisfies $u(\{m\}) \Varangle m$. If $M(u)$ is bounded, then $u[M(u)]$ is a maximal member of $M$.

Proof. If $M(u)$ is bounded then, by the final result of first paragraph of the preceding proof, it has a greatest member $m$, and $u(\{m\}) \leq m$. The restriction on $u$ then requires that $m$ be maximal. But $m \leq u[M(u)]$, so that $u[M(u)]$ is also maximal.

COROLLARY 2. Let $M$ have the property that if $\Lambda \subset N \subseteq M$, and $N$ is bounded, then $N$ has a least upper bound; and let $u$ satisfy the additional restrictions that if $\Lambda \subset N \subseteq M$, and $N$ is bounded but has no greatest member, then $u(N)$ is a least upper bound of $N$; and that if $m, n \in M$ and $m<n$ then $u(\{m\}) \leq$ 
$u(\{n\})$. If there exists $a p \in M$ such that $a \leq p$ and $u(\{p\}) \leq p$ then $M(u)$ is bounded and $u[M(u)]$ is a least such $p$ (cf. [2, p. 83. Th. $\left.\left.\mathrm{II}^{\prime}\right]\right)$.

Proof. Suppose $a \leq p$ and $u(\{p\}) \leq p$. If $m \in M(u)$, not the greatest, and $m \leq p$, then $m^{+}=u(\{m\}) \leq u(\{p\}) \leq p$. If $m \in M(u)$ and has no immediate predecessor in $M(u)$, and $p$ is an upper bound of $M(u)_{m}$, then $m=u\left(M(u)_{m}\right)$ $\leq p$. Hence, by induction, $p$ is an upper bound of $M(u)$. Hence $M(u)$ has a greatest member $m$, with $u(M(u)) \leq m \leq p$ and $u(\{u[M(u)]\}) \leq u(\{m\}) \leq$ $m \leq u[M(u)]$. Consequently, $u[M(u)]$ is a least such $p$.

5. On Zorn's lemma. We give now an alternative proof of Szele's result:

THEOREM 2. The axiom of choice implies Zorn's lemma.

Proof. Let $M$ be ordered by $<, a \in M$, and $f$ a choice - function for the set of all nonempty subsets of $M$. Define $g$ as follows: $g(M)=a$; if $N \subset M$ and either has no least member or coincides with the set of its least members then $g(N)=f(N)$; if $N \subset M$ and has a least member but does not coincide with the set $N_{0}$ of its least members then $g(N)=f\left(N-N_{0}\right)$. Define $u$ as follows: $u(N)=g(\{x \mid x \in M$ and $(y)(y \in N \Longrightarrow y \leq x)\})$. Then $u(\Lambda)=a$ and if $N, P \subseteq M$ and have the same nonempty set of upper bounds then $u(N)=u(P)$. If $m$ is a nonmaximal member of $M$ then the set of upper bounds of $\{m\}$ has a least member $m$, but does not coincide with the set of its least members. Hence $u(\{m\})$ is not a least upper bound of $\{m\}$, and $u(\{m\}) \nmid m$. Since the hypotheses of Theorem 1 and Corollary 1 are satisfied, we see that if $M(u)$ is bounded then $u[M(u)]$ is a maximal member of $M$, and $a \leq u[M(u)]$.

From this, one obtains Zorn's lemma in the strong form (cf. [3, p. 27, Th. 9]): If $M$ is ordered by $<$, and every subset of $M$ which is well-ordered by $<$ has an upper bound, then for each a $\in M$, there is a maximal member $m$ of $M$ such that $a \leq m$.

6. Remarks. Note that only the first paragraph of the proof of Theorem 1 is appealed to in the proof of Theorem 2, and that for this application the hypotheses on $u$ can be simplified to the requirements that $u(\Lambda)=a$ and that if $N$ and $P$ have the same nonempty set of upper bounds then $u(N)=u(P)$.

It may also be worthy of note that, in the first paragraph of the proof of Theorem 1, the transitivity of $<$ was used only in the last sentence. If $<$ is assumed to be antisymmetric (but not necessarily transitive) this sentence might be replaced by: Since < is antisymmetric, we have $m=u[M(u)]$. Up to this point the only properties of $u$ that have been used are that $u(\Lambda)=a$ and that $u(N)$ is an upper bound of $N$. Appealing to the hypothesis that if $N$ and $P$ 
have a common greatest member then $u(N)=u(P)$, we can conclude that $u(\{u[M(u)]\})=u(M(u))$.

Now let an $m \in M$ be called 'weakly maximal' when, for every $n \in M$, it satisfies the following condition: if $p \leq m \Longrightarrow p<n$ for every $p \in M$, then $n<m$. Clearly, a maximal member of $M$ is weakly maximal, and if < is transitive then the converse also holds. If $<$ is antisymmetric then the phrase ' $n<m$ ' can be replaced by ' $n=m$ '. If, in Corollary 1, 'maximal' is replaced by 'weakly maximal' then the resulting statement is true not only if < is transitive but also if it is assumed only to be antisymmetric.

Using the axiom of choice, one can define a function $u$ which satisfies the four conditions referred to in the two preceding paragraphs, as follows: $u(\Lambda)=a$; if $N$ has a greatest member $m$ which is not weakly maximal then

$u(N)=f(\{x \mid x \in M$ and $x \neq m$ and $(y)((y \in M$ and $y \leq m) \Longrightarrow y<x)\})$

if $N$ has a greatest member $m$ which is weakly maximal then $u(N)=m$; if $N$ is bounded and nonempty and has no greatest member then

$$
u(N)=f(\{x \mid x \in M \text { and }(y)(y \in N \Longrightarrow y \leq x)\}) .
$$

Hence we have the following analogue of Zorn's lemma:

If $<$ is an antisymmetric relation defined on $M$, and every subset of $M$ which is well-ordered by $<$ has an upper bound, then, for each $a \in M$, there is a weakly maximal member $m$ of $M$ such that $a \leq m$; that is, $a \leq m$, and if $n \neq m$ then there is a $p$ such that $p \leq m$ and $p \nless \bar{n}$.

7. Addendum. After this paper was submitted to the editors it was brought to the author's attention that certain results similar to those stated in Theorem 1 had recently been published by Bourbaki [7]. He assumes that a set $E$ is given on which there is a transitive and antisymmetric relation $\leq$ and a transformation $f$ of $E$ into itself such that: $x \leq f(x)$ for every $x \in \bar{E}$. In addition $a \in E$. His theorem asserts the existence of a set $A$ which is the least of the sets $P \subseteq E$ such that $a \in P$; if $x \in P$ then $f(x) \in P$; if $\Lambda \subset N \subseteq P$ and $N$ has a least upper bound then this least upper bound belongs to $E$. The theorem also asserts that $\leq$ is connected on $A$. Bourbaki later proves that $A$ is well ordered by $\leq$, and that $A$ has a greatest member if and only if it has a least upper bound.

It is interesting to note that Bourbaki's results follow from Theorem 1. For the proof, let $a_{\infty}$ be anything not in $E$, and let $M$ be $E \cup\left\{a_{\infty}\right\}$. Extend the relation $\leq$ and the function $f$ to $M$ by requiring that $x<a_{\infty}$ for every $x \in E$, and that $f\left(a_{\infty}\right)=a_{\infty}$. Define $u$ as follows: $u(\Lambda)=a$; if $\Lambda \subset N \subseteq M$ and is bounded, 
there are three cases: If $N$ has a least upper bound $m$ but no greatest member, then $u(N)=m$; if $N$ has a greatest member $n$ then $u(N)=f(n)$; if $N$ has no least upper bound then $u(N)=a_{\infty}$. With this definition it is easily shown that $M(u)-\left\{a_{\infty}\right\}$ is the desired set $A$.

\section{REFERENCES}

1. G. Hessenberg, Kettentheorie und Wohlordnung, J. Reine Angew. Math. 135 (1909), $81-133$.

2. C. Kuratowski, Une méthode d'élimination des nombres transfinis des raisonnements mathématiques, Fund. Math. 3 (1922), 76-108.

3. A. Milgram, Partially ordered sets, separating systems and inductiveness, Rep. Math. Colloquium, Second Series, Issue 1 (1939) 18-30.

4. T. Szele, On Zorn's lemma, Publ. Math. Debrecen 1 (1950), 254-257.

5. E. Zermelo, Beweis dass jede Menge wohlgeordnet werden kann, Math. Ann. 59 (1904), 514-516.

6. , Neuer Beweis für die Wohlordnung, Math. Ann. 65(1908), 107-128.

7. N. Bourbaki, Sur le théorème de Zorn, Arch. Math. 2 (1949) 434-437.

\section{UNIVERSITY OF ILLINOIS}




\title{
PACIFIC JOURNAL OF MATHEMATICS
}

\section{EDITORS}

\section{R. M. RobINSON \\ University of California \\ Berkeley 4, California}

\author{
*R. P. Dilwor TH \\ California Institute of Technology \\ Pasadena 4, California
}

\author{
E. F. BECKENBACH, Managing Editor \\ University of California \\ Los Angeles 24, California
}

${ }^{*}$ During the absence of Herbert Busemann in 1952.

\section{ASSOCIATE EDITORS}

$\begin{array}{llll}\text { R. P. DILWORTH } & \text { P. R. HALMOS } & \text { B } \emptyset \text { RGE JESSEN } & \text { J. J. STOKER } \\ \text { HERBERT FEDERER } & \text { HEINZ HOPF } & \text { PAUL LÉVY } & \text { E. G. STRAUS } \\ \text { MARSHALL HALL } & \text { R. D. JAMES } & \text { GEORGE PÓLYA } & \text { KÖSAKU YOSIDA }\end{array}$

\section{SPONSORS}

UNIVERSITY OF BRITISH COLUMBIA

CALIFORNIA INSTITUTE OF TECHNOLOGY

U NIVERSITY OF CALIFORNIA, BERKELEY

UNIVERSITY OF CALIFORNIA, DAVIS

UNIVERSITY OF CALIFORNIA, LOS ANGELES

UNIVERSITY OF CALIFORNIA, SANTA BARBARA

OREGON STATE COLLEGE

UNIVERSITY OF OREGON

\author{
UNIVERSITY OF SOUTHERN CALIFORNIA \\ STANFORD UNIVERSITY \\ WASHINGTON STATE COLLEGE \\ UNIVERSITY OF WASHINGTON \\ AMERICAN MATHEMATICAL SOCIETY \\ NATIONAL BUREAU OF STANDARDS, \\ INSTITUTE FOR NUMERICAL ANALYSIS
}

Mathematical papers intended for publication in the Pacific Journal of Miathematics should be typewritten (double spaced), and the author should keep a complete copy. Manuscripts may be sent to any of the editors. All other communications to the editors should be addressed to the managing editor, E. F. Beckenbach, at the address given above.

Authors are entitled to receive 100 free reprints of their published papers and may obtain additional copies at cost.

The Pacific Journal of Mathematics is published quarterly, in March, June, September, and December, by the University of California, Berkeley 4, California. The price per volume (4 numbers) is $\$ 8.00$; single issues, $\$ 2.50$. Special price to individual faculty members of supporting institutions and to individual members of the American Mathematical Society: $\$ 4.00$ per volume; single issues, $\$ 1.25$.

Subscriptions, orders for back numbers, and changes of address should be sent to the publishers, University of California Press, Berkeley 4, California.

Printed at Ann Arbor, Michigan. Entered as second class matter at the Post Office, Berkeley, California.

\section{UNIVERSITY OF CALIFORNIA PRESS • BERKELEY AND LOS ANGELES}




\section{Pacific Journal of Mathematics}

\section{Vol. 2, No. $3 \quad$ March, 1952}

Lars V. Ahlfors, Remarks on the Neumann-Poincaré integral equation .... 271

Leonard P. Burton, Oscillation theorems for the solutions of linear, nonhomogeneous, second-order differential systems ............ 281

Paul Civin, Multiplicative closure and the Walsh functions . . . . . . . . . . . 291

James Michael Gardner Fell and Alfred Tarski, On algebras whose factor algebras are Boolean .................................. 297

Paul Joseph Kelly and Lowell J. Paige, Symmetric perpendicularity in Hilbert geometries .................................. 319

G. Kurepa, On a characteristic property of finite sets .............. 323

Joseph Lehner, A diophantine property of the Fuchsian groups ......... 327

Donald Alan Norton, Groups of orthogonal row-latin squares ........... 335

R. S. Phillips, On the generation of semigroups of linear operators ....... 343

G. Piranian, Uniformly accessible Jordan curves through large sets of relative harmonic measure zero ........................ 371

C. T. Rajagopal, Note on some Tauberian theorems of $O . S z \tilde{A} ; s z \ldots \ldots \ldots 377$

Halsey Lawrence Royden, Jr., A modification of the Neumann-Poincaré method for multiply connected regions .................... 385

George H. Seifert, A third order irregular boundary value problem and the associated series ...................................... 395

Herbert E. Vaughan, Well-ordered subsets and maximal members of ordered sets....

Hans F. Weinberger, An optimum problem in the Weinstein method for eigenvalues.

Shigeki Yano, Note on Fourier analysis. XXXI. Cesàro summability of Fourier series. 